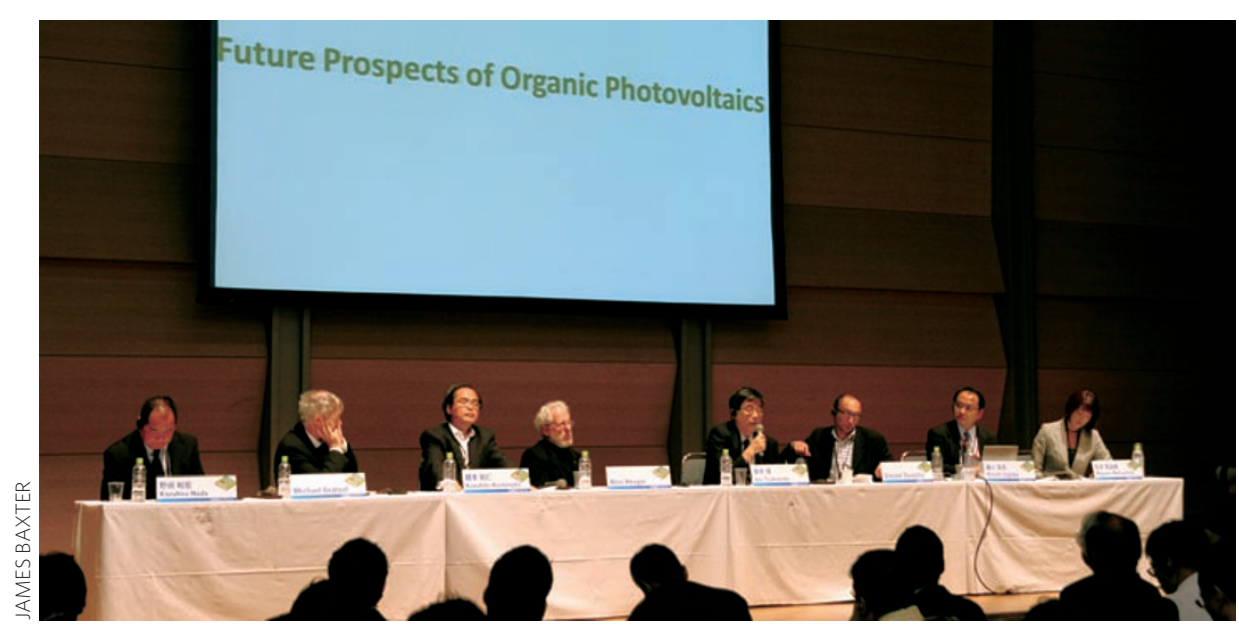

Experts sharing their thoughts on the future prospects of organic photovoltaics during the panel discussion session.

potentially lightweight, flexible, rugged, insensitive to solar incidence angle and can be produced by roll-to-roll manufacturing techniques in large quantities.

Based on ultrafast photo-induced electron transfer in bulk-heterojunction nanomaterials, the power conversion efficiency of polymer solar cells has now reached $\sim 8 \%$, and this can be pushed further according to Heeger. Although currently the absorption band doesn't cover wavelengths longer than $650 \mathrm{~nm}$ and therefore matches poorly with the solar spectrum, Heeger explained that this can be solved by synthesizing new macromolecules with electronic structures that yield absorption spectra better matched to the solar spectrum. Additionally, through further improvements such as adding an optical spacer layer, optimizing the electrochemistry of the semiconducting polymers or using a tandem-cell configuration, the power conversion efficiency of plastic solar cells could reach an efficiency approaching that of existing inorganic solar cells. "Plastic solar cells could become a very important contribution towards a renewable energy economy," Heeger concluded.

A report that summarizes the event, including opinions from the conference, a round-up from the exhibition and several interviews with leading experts in the field of photovoltaics, will be published online and in print as a supplement in early 2011. $\square$

Rachel Won is at Nature Photonics, Chiyoda Building, 2-37 Ichigayatamachi, Shinjuku-ku, Tokyo 162-0843, Japan.

e-mail:r.won@natureasia.com

\title{
NANOMETROLOGY
}

\section{Diffraction rules}

Fast, precise and stable nanopositioning and metrology are critical for the development of nanoscale structures, particularly in the semiconductor industry. Present measurement techniques that offer high precision are typically slow or lack long-range travel and reproducibility. For example, scanning-tip approaches often have trouble accurately returning to a given location after moving distances of just tens of micrometres. Alternative approaches also have disadvantages, such as limited bandwidth in the case of optical encoder-based systems, or poor temporal stability.

Now, Norimasa Yoshimizu and colleagues from Cornell University in the USA have reported a highly precise form of metrology that uses an optical ruler imaging system based on diffraction and signals processing (Opt. Express 18, 20827-20828; 2010). The technique involves cross-correlating a feature-rich diffraction pattern projected onto silicon wafers using an atomically stabilized laser beam, and enables metrology with a spatial precision of $17.2 \mathrm{~nm}$ across a

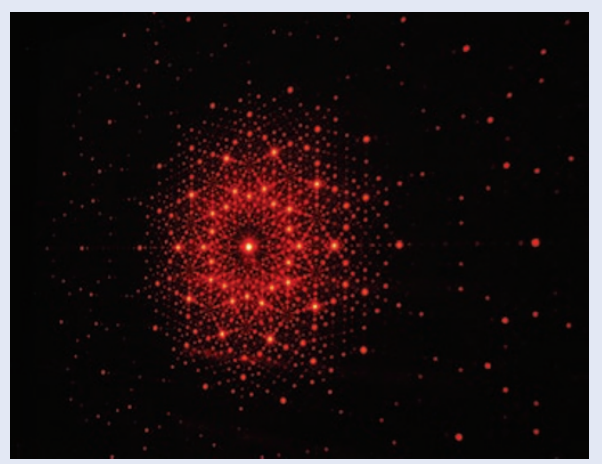

displacements with high precision over a large working range and with long-term stability. According to Yoshimizu, one of the main challenges was determining how best to create a rich diffraction pattern.

"We're all familiar with diffraction from periodic structures - Young's double slit experiments, for example - but these provide very regular diffraction patterns with hardly any features or random patterns," Yoshimizu commented. "Quasiperiodicity is an interesting way of generating diffraction patterns with sharp and dense features."

wafer several inches in size with a long-term stability of around six hours.

One key to achieving this precision is the use of atomic vapour to stabilize the laser frequency. The laser beam is then diffracted from a microfabricated thin metal film patterned with holes in a quasiperiodic manner. The resulting dense asymmetric diffraction pattern illuminates the wafer sample and functions as a visible optical ruler. Using a camera and digital signals processing, the team were able to use the pattern to measure nanoscale
The team is now working towards further improving the precision and working range of their technique, as well as the integration of atomic force microscope probes with active CMOS sensors to image the diffraction pattern. There is hope that this cross-correlation technique could be used for high-precision displacement measurements in rotation sensors and accelerometers, as well as for applications in biological imaging.

DAVID PILE 